\title{
Du sang à l'écriture. Les pratiques rituelles des Sora, une tribu du centre-est de l'Inde
}

Doctorat en anthropologie sociale, sous la direction de Marine Carrin, École des hautes études en sciences sociales (EHESS), soutenu le 12 décembre 2011.

Cécile Guillaume-Pey

\section{(2) OpenEdition}

Édition électronique

URL : http://journals.openedition.org/assr/24583

DOI : $10.4000 /$ assr.24583

ISSN : $1777-5825$

Éditeur

Éditions de l'EHESS

Édition imprimée

Date de publication : 30 décembre 2012

Pagination : 309-358

ISSN : 0335-5985

Référence électronique

Cécile Guillaume-Pey, « Du sang à l'écriture. Les pratiques rituelles des Sora, une tribu du centre-est de I'Inde », Archives de sciences sociales des religions [En ligne], 160 | octobre-décembre 2012, mis en ligne le 14 mars 2013, consulté le 03 mai 2019. URL : http://journals.openedition.org/assr/24583

Ce document a été généré automatiquement le 3 mai 2019.

(c) Archives de sciences sociales des religions 


\section{Du sang à l'écriture. Les pratiques rituelles des Sora, une tribu du centre- est de l'Inde}

Doctorat en anthropologie sociale, sous la direction de Marine Carrin, École des hautes études en sciences sociales (EHESS), soutenu le 12 décembre 2011.

\section{Cécile Guillaume-Pey}

1 Ce travail porte sur les pratiques religieuses des Sora, un groupe tribal du centre-est de l'Inde parlant une langue austro-asiatique. Il est le fruit d'une enquête de terrain de neuf mois principalement menée dans des villages sora du nord de l'Andhra Pradesh, lesquels ont été fort peu étudiés en comparaison de ceux de l'Orissa. Les Sora, qui se démarquent d'un point de vue linguistique des autres groupes locaux, ne constituent en aucun cas une population totalement isolée de la société des castes. En ce qui concerne leurs pratiques rituelles, ils présentent certains traits qui leur sont propres, mais on observe aussi de nombreux points de rencontre avec la religion dominante, dont certains se revendiquent d'ailleurs. La dialectique de l'emprunt et du rejet se décline en fait différemment au sein d'un même groupe. Parmi les Sora des villages étudiés, trois formes d'affiliation religieuse coexistent. Certains se réclament d'une grande religion: l'hindouisme ou le christianisme. D'autres adhèrent à un culte qui leur est propre, nommé « Matharvanam », et opposent leurs pratiques rituelles à celles des castes comme à celles des Sora hindous et chrétiens. Ces derniers rendent un culte à une écriture inventée dans les années 1930 pour transcrire leur langue. Si ces trois ensembles religieux comprennent chacun des espaces cultuels, des officiants, et un panthéon qui leur sont propres, des connexions s'établissent entre des acteurs affiliés à différents cultes, en particulier dans le contexte des rites funéraires et celui des rites de cure. Il arrive en outre que derrière les affiliations revendiquées et les oppositions apparentes, on observe une certaine labilité au niveau des représentations et que les personnes, selon le contexte, jonglent entre des pratiques rituelles très différentes. 
2 Pour rendre compte de la coexistence de ces formes cultuelles hétérogènes élaborées au confluent d'influences multiples, l'analyse est conduite à plusieurs niveaux. Il s'agit d'une part d'étudier les modalités de construction des identités religieuses à l'échelle microlocale. Et d'autre part de mettre en lumière les points de rupture et les continuités existant au niveau religieux entre les Sora affiliés à différents cultes et d'autres groupes sociaux: des castes, de bas statut en particulier, et des groupes tribaux, dont certains, à l'instar des Sora, parlent une langue austro-asiatique et célèbrent des rites de secondes funérailles marqués par l'érection de stèles pour leurs ancêtres. Enfin, la comparaison entre les pratiques religieuses des Sora du nord de l'Andhra Pradesh et celles de leurs voisins du sud de l'Orissa, sur lesquels portent les principaux travaux consacrés aux Sora (V. Elwin, 1955 ; C. Petit, 1974 ; P. Vitebsky, 1993)1', permet de s'interroger sur l'unité d'un groupe tribal de l'Inde disséminé entre différents États.

3 La thèse comporte cinq parties : la première vise tout d'abord à éclairer la notion de « tribu » tel qu'elle est utilisée en Inde pour circonscrire certaines populations (Scheduled Tribes). Comment certains groupes sont identifiés comme " tribaux »? Quel rôle le critère religieux joue-t-il dans la constitution de cette catégorie et dans la définition des contours d'une identité «tribale» en opposition à celle de la caste depuis l'époque coloniale? Cette partie présente ensuite le groupe étudié : son histoire, la nature des relations nouées avec divers groupes locaux - castes et tribus - et les principaux traits de son organisation sociale. L'absence d'organisation clanique, qui distingue les Sora des autres groupes tribaux de langue austro-asiatique, est soulignée puisqu'elle permet d'éclairer les variations évoquées par la suite dans le champ religieux, et plus particulièrement concernant la définition des unités de culte.

4 La deuxième partie traite du panthéon des Sora hindous et de son ancrage spatial. Après s'être interrogé sur les principes classificatoires permettant d'articuler le divin, trois classes de puissances sont distinguées: les nyonan, divinités chtoniennes autogénérées fondamentalement ambivalentes; les défunts récents, kulba; et les ancêtres, elda, pacifiés par les rites funéraires. Les rapports entre les Sora et leur environnement physique et social sont médiatisés par l'intermédiaire de ces puissances avec lesquelles ils établissent des relations de type contractuel. À la différence de ces classes divines, étroitement liées au territoire villageois, et qui jouent un rôle prépondérant dans les rituels, les dieux hindous sont associés aux temples situés au cœur de l'espace urbain et occupent paradoxalement une place relativement marginale au sein des pratiques religieuses des Sora qui se réclament de l'hindouisme.

5 Dans la partie suivante, il est question des unités de culte associées aux principaux espaces rituels où les différentes catégories divines du panthéon sora sont présentifiées. Ces lieux présentent des similarités importantes avec ceux observés dans d'autres groupes de langue austro-asiatique de l'Inde, les sites funéraires en particulier. Il existe néanmoins des différences notoires en ce qui concerne les unités sociales qui en ont l'usage, ce qui permet de souligner la spécificité du groupe étudié, non seulement par rapport à d'autres groupes tribaux, mais également par rapport aux Sora de la région voisine. Ainsi, du point de vue de la définition des unités de culte, l'appartenance territoriale prime sur l'appartenance lignagère, phénomène éclairé par l'examen des relations entre le pouvoir, la parenté et le territoire, lesquelles se déclinent différemment pour les Sora de l'Orissa et ceux de l'Andhra Pradesh.

6 Les rites de cure, qui occupent une place centrale au sein des pratiques religieuses des Sora, font l'objet de la quatrième partie. L'examen de ces rituels, organisés autour de la 
possession et du sacrifice, permet d'une part d'aborder les interactions en jeu entre divers types de spécialistes religieux mobilisés dans ce contexte - dont le mode d'action respectif fait l'objet d'un chapitre particulier - et des individus ordinaires, sans lesquels les premiers ne sauraient mener à bien le «travail» rituel. L'étude de ce champ particulier permet d'autre part de s'interroger sur les modalités et les enjeux du changement d'affiliation religieuse. Dans le contexte des rites de cure, en effet, on observe des rapprochements avec l'hindouisme, des conversions au christianisme et divers cas de bricolage ou de « jonglage » entre différentes formes cultuelles.

7 La dernière partie est dédiée aux adeptes du mouvement de Matharvanam, qui, à la différence des Sora se définissant comme hindous, ont choisi d'ajuster leurs pratiques religieuses sur celles des castes supérieures tout en refusant de se considérer comme hindous. Ils vénèrent une écriture révélée, considérée à la fois comme l'incarnation des puissances du panthéon sora et de Jagannath, avatar du dieu hindou Vishnu symbolisant le pouvoir royal et celui des brahmanes. Toujours en quête de nouvelles réappropriations visant des figures divines puisées dans différents registres, ces adeptes s'évertuent à se démarquer de ceux qui ne se sont pas emparés du support écrit, notamment par une condamnation sans appel du sacrifice sanglant. Il s'agit alors de s'interroger sur les clivages sociaux mis en jeu par l'écriture révélée : si Matharvanam permet d'unifier une population tribale disséminée entre différents États, au niveau local, il génère un processus de différenciation entre les adeptes du mouvement et les Sora affiliés à une autre religion.

Ce travail cherche ainsi à rendre compte d'un paysage religieux complexe et mouvant, où les pratiques rituelles observées sont loin d'être homogènes - et qu'on ne saurait dès lors opposer, ou comparer en bloc, à celles d'autres groupes sociaux, castes de statut divers ou tribus -, en prenant en compte les affiliations religieuses revendiquées, qui parfois correspondent peu aux pratiques rituelles effectives. La revendication d'une identité hindoue, la conversion au christianisme, tout comme l'adhésion au culte d'une écriture révélée, sont autant de processus qui permettent de s'interroger sur la manière dont le religieux produit de la différence au niveau microlocal dans un groupe tribal relativement homogène du point de vue social, et offre alors à ses membres diverses manières de se situer par rapport à la société dominante.

\section{NOTES}

1. V. Elwin, The Religion of an Indian Tribe, London, Oxford University Press, 1955 ; C. Petit, La Culture matérielle des Saora, Thèse de doctorat, Université de Paris III (non publiée), 1974 ; P. Vitebsky, Dialogues with the Dead, The discussion of Mortality among the Sora of Eastern India, Cambridge, Cambridge University Press, 1993. 


\section{AUTEUR}

CÉCILE GUILLAUME-PEY

heyokcha@hotmail.com 